\title{
Why the Big Bang Singularity does not Help the Kalām Cosmological Argument for Theism
}

September 8, 2007

\begin{abstract}
The Big Bang singularity provides little or no evidence for creation in the finite past and hence for theism. Whether one dismisses singularities or takes them seriously, physics licenses no first moment of (space-)time. A physical theory might lack a metric or any other notion of finite length for curves, so a general notion of "beginning" must involve a first moment. The analogy between the Big Bang singularity and stellar gravitational collapse suggests that a Creator is required in the first case only if a Destroyer is needed in the second. The need for and progress in quantum gravity and the underdetermination of theories by data make it difficult to take singularities seriously.
\end{abstract}

\section{Introduction}

The epistemic status of theistic belief continues to be of interest. The modern western intellectual climate being skeptical about divine revelation and the supernatural in general, theists seem to be well served if good theistic arguments from natural philosophy are available. One of the more attractive theistic "proofs," given current science, employs the Big Bang singularity to show that the universe began to exist. This theistic apologetic strategy proceeds sometimes by explicit use of the formal Kalām cosmological argument, sometimes less formally. Whatever the details, this type of argument has commended itself to not a few reputable physicists and other scientists and philosophers, as well as at least one Pope, Pius XII [Pius XII, 1952, McMullin, 1981a]. In the 1960s Engelbert Schücking announced that "[a]lthough some cosmologists might still unconsciously project theological pictures into their science, we have been able to scare most of the ministers out of cosmology by a straightforward application of tensor calculus." [Schücking, 1967] 
Schücking notwithstanding, significant and visible efforts continue to be made to support theism via the Big Bang singularity. Evidently some atheists have agreed that Big Bang cosmology supports theism, and so have doubted Big Bang cosmology:

Perhaps the best argument in favour of the thesis that the Big Bang supports theism is the obvious unease with which it is greeted by some atheist physicists. At times this has led to scientific ideas ... being advanced with a tenacity which so exceeds their intrinsic worth that one can only suspect the operation of psychological forces lying very much deeper than the usual academic desire of a theorist to support his/her theory. [Isham, 1997, p. 378].

While some theists and some atheists hold that the singularity supports theism, members of both groups have criticized the argument as well. However, atheistic critics not infrequently mix with their good points various unhelpful moves such as denying ex $n i$ hilo, nihil fit and thus perhaps ceding the rational high ground, introducing premises that strike the theistic apologist as question-begging, or writing in a tone suggesting that rejection of the theistic conclusion plays an undue role in motivating the rejection of the argument. Judging by the lack of progress in some of the literature, the result is a stalemate. Quentin Smith, who has played a role in critiquing the singularity argument for theism, has a different but also negative evaluation of the overall response to the theistic argument from the Big Bang singularity [Smith, 1992]: "The response of atheists and agnostics ... has been comparatively weak, indeed, almost invisible."

Thus there is room, I take it, for a critique of the argument from the Big Bang singularity to theism that strives to convince even the Big Bang singularity argument's proponents, not just the indifferent and the already opposed. If an argument is bad, there is value in showing its badness even to its proponents, if possible. Such a critique will require a brief discussion of the doctrine of creation. It will also involve some concessions to the Big Bang theistic apologist that might otherwise seem overly generous. Some arguments will be aimed at persuading the theistic apologist that the Big Bang singularity is an unpromising strategy.

The critique presented here also has some novel elements, I believe, which should be of direct interest to nontheists and fence-sitters as well. A reductio ad absurdum strategy deployed below leads to the conclusion that the Big Bang singularity implies a theistic Creator only if stellar gravitational collapse to a black hole implies a theistic Destroyer. The Bach-Weyl theory of gravity shows that a physical theory need not even define the length of a curve; thus only a topological notion of "beginning" in terms of a first moment is available if the Kalām argument is intended to yield a necessarily nonvacuous necessary 
truth. But a first moment is easy to avoid, as will appear below.

It should be emphasized that the point at issue is not primarily the soundness of the Kalām cosmological argument, but rather its dialectical effectiveness. A theist might think that the Kalām argument is sound and accept the critique made here, by holding that the key controversial premise of the Kalām argument ought to be accepted not on the grounds of Big Bang cosmology, but only on the basis of divine revelation. The medieval debate about Aristotle's doctrine of the eternity of the world provides a very helpful precedent.

\section{The Doctrine of Creation and Its Warrant}

Historically, the doctrine of creation has been basically common property among Judaism, Christianity and Islam. While there have been some differing views about the doctrine, the differences have little to do with the differences between these Abrahamic religions. A consensus arose that, pace Plato's Timaeus, creation was ex nihilo: God brought (or timelessly brings) all other things into existence and does so without using pre-existing materials. Furthermore, God creates the world voluntarily, as a result of willing rather than as an unwilled necessary emanation. Besides the initial creation ex nihilo "in the beginning," there is some sort of ongoing sustenance, preservation, continuing creation or the like, by which God keeps the world in existence and without which it would cease to exist [Quinn, 1993].

Given creation ex nihilo, a secondary question is whether God created the world in the finite past or eternally. We may speak of "creation in time" if the universe is finitely old and had a first moment of existence. (If the universe is finitely old but had no first moment, then it is unclear whether creation in time or eternal creation is a more appropriate term, especially on account of conventionality worries to be discussed below.) At times some have denied the initial creation in favor of God's eternally creating the universe. In medieval times this denial occurred with Islamic philosophers such as Avicenna and Averroes and with the very Aristotelian Averroists in western Christendom. This "eternal creation" view was defeated by orthodoxy defending creation in time, such as the Fourth Lateran Council in 1215 and the condemnations by Bishop Tempier of Paris in 1277.

Advocates of creation in time have disagreed whether the finite age of the universe could be known by reason or only by faith [Thijssen, 1998]. In response to Aristotelian philosophical arguments for an eternal universe, Moses Maimonides and Thomas Aquinas 
both held such arguments to be inconclusive. Both also held the philosophical arguments of their days in favor of a finite age to be inconclusive. Thus the issue was left to be resolved by Scripture, which favored finite age [Hyman and Walsh, 1973]. Bonaventure, by contrast, took the finite age of the universe to be demonstrable philosophically. This distinction is closely analogous to the issue addressed in this paper. Those who argue for theism from the Big Bang singularity follow Bonaventure in spirit. I will argue that theists who affirm creation in time ought to hold, with Maimonides and Aquinas in spirit, that creation in time is known by faith rather than by natural philosophy - or at least, not from the singularity in Big Bang cosmology.

I will comment briefly on William Lane Craig and Paul Copan's a priori arguments for a finite past. Regarding the supposed impossibility of a traversing an actual infinity [Craig, 1979, Copan and Craig, 2004], one might object to the treatment of infinity [Morriston, 2003]. Regarding the possibility of the existence of an actual infinity, I cannot find anything absurd in Hilbert's hotel, which has as many rooms as there are positive integers. It appears that Craig's objections are due in no small part to the widespread claim that the concept of size is fully captured by the notion of cardinality. But one might well accept the possibility of actual infinities, such as Hilbert's hotel, while denying that cardinality exhausts the notion of sameness of size. It is also difficult to regard as omnipotent a God who could not create Hilbert's hotel. These comments are incidental to the thrust of this paper, however. If creation in time could be known a priori, then arguments from physical cosmology would be largely redundant. For the sake of argument I therefore assume that a finite past is metaphysically possible.

In modern theology, the initial creation was downplayed Friedrich Schleiermacher as not necessitated by his controlling principle of the feeling of absolute dependence [Russell, 1996b]. Moreover, he took the doctrine of the initial creation event to depend on the Genesis creation account, which he took to be the product of a mythological time [Copan and Craig, 2004, pp. 150, 151]. Quite a few modern scholars have felt free to discard or hold lightly the initial creation event, so eternal creation is now regarded as attractive in some circles [Russell, 1996a]. According to John Polkinghorne, "[t]heology is concerned with ontological origin and not with temporal beginning. The idea of creation has no special stake in a datable start to the universe." [Polkinghorne, 1994] Paul Helm has agreed [Helm, 1997]. The question has also arisen whether Genesis 1:1 actually asserts a beginning or not, but the traditional interpretation still certainly has defenders [Copan and Craig, 2004]. It should go without saying that the interpretation of a text is a distinct issue from the correctness of its assertions. 
If a universe is created, is it eternal, finitely old but lacking a first moment, or finitely old with a first moment? The universe's having a first moment entails its being finitely old (unless one introduces a point at infinity, which seems purely formal), but the converse entailment might fail. If time is continuous, then the world's being finitely old does not entail its having a first moment. At least prima facie it seems that a universe with a first moment is the sort most confirmatory of theism [McMullin, 1981b], while an eternal universe is the least helpful. If so, then eternal creation might not be the best view for religious theists. Regarding the two extreme cases, Aquinas held something along these lines, as Ernan McMullin discusses:

If the universe began at a point of time, would this give stronger support to the claim that a Creator is needed than if the universe always existed? Aquinas argued that in a sense it would, even though he was insistent that a universe which had always existed would equally need a Creator to sustain it. But creation in time rather than from eternity makes the work of God's power more evident, Aquinas says, because an agent displays the more power in acting, the more removed from act is the potency acted upon. And in creation in time there is no potency of any kind to work on. This of itself immediately shows the infinity of power required to summon a universe into act. [McMullin, 1981a, p. 39]

A first moment appears to convey three advantages on the doctrine of creation over mere finite age. First, it is more obvious that a finitely old universe with a first moment requires an external Cause than that a finitely old universe in which every moment is preceded by an earlier one does. In the latter case, one might be tempted to think that the present is fully explained by the past within the history of the universe, so nothing external is required [Grünbaum, 1989]. Second, having a first moment is a topological notion, not a metrical one, and so escapes conventionality worries [Levy-Leblond, 1990, Misner, 1969, Agnese and Wataghin, 1971, McMullin, 1981a, Grünbaum, 1989] about temporal remetrization of a finite past to an infinite one and the conventionality of the metric [Grünbaum, 1973]. Conventionalist questions about the significance of the difference finite $v s$. infinite seem to be due to E. A. Milne [Kragh, 2004, p. 209][McMullin, 1981a]. Metrical conventionality becomes an especially serious worry in physical theories containing multiple metrics. Scalar-tensor theories are perhaps the best known locus for the question "Which metric is the physical metric?" [Weinstein, 1996, Magnano and Sokolowski, 1994, Kaloper and Olive, 1998, Santiago and Silbergleit, 2000]. While this question seems not to need an answer for most 
purposes (such as those involving only the field equations), questions of singularities, boundary conditions, positive energy and quantization give that question a bit more urgency [Kaloper and Olive, 1998, Santiago and Silbergleit, 2000, Faraoni and Nadeau, 2007, Sotiriou et al., 2007, Catena et al., 2006]. Theories with multiple metrics might have different types of matter coupling to gravity in different ways; then perhaps one metric might yield finite age, but another infinite age, in which case there seems to be no answer to the question "how old is the universe?", even if the options at hand are merely "finite" and "infinite." It turns out that the actual universe probably does not behave in accord with a scalar-tensor theory, given the empirical confirmation of the various principles of equivalence in gravity [Will, 1993], which make it difficult for theories empirically distinguishable from GTR in weak or moderate gravitational fields to be empirically viable. But a doctrine of creation needs to be modally rich enough to accommodate the possibility of God's creating worlds with physical laws without a unique or preferred metrical structure to license an answer of "finite" or of "infinite." Third, a first moment, being topological rather than metrical, is well defined even in the absence of a metric. Bach-Weyl conformal gravity [Fiedler and Schimming, 1980, Schimming and Schmidt, 1990, Kazanas and Mannheim, 1991, Dzhunushaliev and Schmidt, 2000], in the absence of matter or with some types of matter, employs only the conformal part $\hat{g}_{\mu \nu}$ of a metric, a tensor density with determinant -1 . Given a metric $g_{\mu \nu}$, one can obtain its conformal part using $\hat{g}_{\mu \nu}=g_{\mu \nu}\left(-\operatorname{det} g_{\alpha \beta}\right)^{-\frac{1}{4}}$ in four space-time dimensions. Not having a metric, the Bach-Weyl theory takes $\hat{g}_{\mu \nu}$ as primitive and does not assign lengths to curves. ${ }^{1}$ While light-like (null) geodesics are well defined in the Bach-Weyl theory, their affine parametrization is not [Wald, 1984, p. 446], so even light-like (null) geodesics provide no help in defining finite age. By contrast Copan and Craig simply take for granted the existence, and perhaps the uniqueness, of the metric for timelike curves in setting up the Kalām argument [Copan and Craig, 2004, p. 199] (here presented a bit differently from the version quoted below):

\footnotetext{
${ }^{1}$ The Lagrangian density of the Bach-Weyl tensor is the square of the Weyl curvature tensor $C_{\rho \sigma \nu}{ }^{\mu}$. The Weyl tensor in its natural habitat is a $(1,3)$ tensor and is a concomitant of the conformal metric density without the determinant $g={ }_{\operatorname{def}} \operatorname{det}\left(g_{\alpha \beta}\right)$ [Anderson, 1967], so it is itself conformally invariant [Wald, 1984]. Thus the Lagrangian density is (up to a constant factor) $\hat{g}^{\rho \alpha} \hat{g}^{\sigma \beta} C_{\rho \sigma \nu}{ }^{\mu} C_{\alpha \beta \mu}{ }^{\nu}$. The absence of $g$ has been made manifest by the use of the inverse conformal metric tensor density $\hat{g}^{\mu \nu}$, which has density weight $\frac{1}{2}$, and the primordial $(1,3)$ form of the Weyl tensor. Much like the Maxwell electromagnetic kinetic term $\hat{g}^{\mu \alpha} \hat{g}^{\nu \beta} F_{\mu \nu} F_{\alpha \beta}$, this Bach Lagrangian density is a weight 1 scalar density, as is required for a coordinate-invariant stationary action principle, without $g$ and so is manifestly conformally invariant.
} 
To assess the truth of the premise [that the temporal series of past, physical events is not beginningless], it will be helpful to define some terms... . In order that all the events comprising the temporal series of past events be of equal duration, we arbitrarily stipulate some event as our standard... . By a "beginning," one means a first standard event. It is therefore not relevant whether the temporal series had a beginning point (a first temporal instant).

Copan and Craig presumably take their Kalām argument to express a necessary truth that applies nonvacuously to all possible physical theories, but their criterion for a beginning is meaningless for the Bach-Weyl theory. It might be ambiguous for scalar tensor theories, which is also somewhat worrisome. Given that neither existence nor uniqueness of a metric (for timelike curves) holds necessarily, the natural move is to adopt a topological rather than metrical notion of beginning. Thus a first moment $i s$ the point that needs to be addressed. However, to adopt a first moment as the criterion for a beginning is to admit defeat, as far as arguing from the singularity to theism is concerned, because the two plausible moves relative to contemporary cosmology (viz. taking space-time to contain only points 'after' the singularity or invoking some perhaps presently unknown theory that resolves the singularity) both lack a first moment. Given the demonstrable contingency of the existence and uniqueness of a metric, it is unclear why finite metrical age is so important even for GTR. As it turns out, the Fourth Lateran Council in 1215 made it a doctrine of faith for Roman Catholics that the world had a temporal beginning, and even escapes the conventionalist worry by positing a first moment [McMullin, 1981a, pp. 29, 54, 55]. The council's definition is topological rather than metrical in character, and so withstands the mathematical advances of the last 800-odd years. Important for present purposes is the fact that this conciliar conclusion is an ostensible deliverance of faith rather than reason.

As McMullin and Aquinas observe, creation in time does have some theological advantages over eternal creation, even apart from adherence to the relevant part of the Genesis account. It would be especially convenient for theists if modern cosmology had provided strong support for creation in time, for both purely theological reasons and for providing an argument for theism along the Kalām lines. On the other hand, the epistemology of creation, at least for the author of the letter to the Hebrews in the New Testament, might favor Aquinas rather than Bonaventure. The key passage is Hebrews 11:3: "By faith we understand that the worlds were prepared by the word of God, so that what is seen was not made out of things which are visible." (NASB) Perhaps Christians should expect that philosophical or scientific demonstrations for creation in time will fail. Whether such an 
expectation is licensed or not, I will argue that the Big Bang singularity fails to provide good evidence for creation in time and hence theism.

\section{Modern Cosmology and Creation}

Modern physical cosmology is a rather effective framework for unifying and explaining a wide variety of astronomical observations in a framework provided by well-confirmed physical laws that hold here and now. Roughly speaking, one assumes a RobertsonWalker spacetime metric satisfying Einstein's gravitational field equations. This metric is spatially homogeneous and isotropic, meaning that every point in space is alike at a given moment of time, and every direction is also. Clearly this is an idealization. Data from the present, especially the fact that luminous objects' redshifts are larger for more distant objects, indicate that the universe is expanding (modulo conventional redescription, which might allow depiction of all objects as shrinking). The dynamics of general relativity, with standard kinds of matter, lets one extrapolate back to an earlier hot dense phase, during which time plausibly the observed cosmic abundances of light elements were produced. One theoretical problem that frequently has been neglected is the "averaging problem," the need to average Einstein's equations over cosmic distances in order to find equations for the cosmic parameters [Ellis, 1984, Buchert and Carfora, 2002, Coley and Pelavas, 2006]. The analogous procedure for electromagnetism in a medium is well-known and comparatively simple due to the linearity of Maxwell's equations. For Einstein's equations, the dynamics of the average bears a complicated relation to the average of the dynamics, so to speak, due to nonlinearities. Experimentally, since the late 1990s it has appeared that the cosmic expansion is accelerating. However, some contend that paying attention to the averaging problem might help to resolve the apparent phenomenon of accelerating expansion, for which "dark energy" has been posited [Buchert, 2007] as an explanation or at least a name. It has also been argued that part of the persuasiveness of the acceleration of the cosmic expansion is an artifact of conventional choices of statistical variables [Cattoen and Visser, 2007]. Little turns on these issues for my purposes, because using the most current version of Big Bang cosmology does nothing to strengthen the Big Bang singularity argument for creation and time and hence theism. The same could be said for inflation, which John Earman and Jesus Mosterin have examined critically [Earman and Mosterin, 1999]. I will freely ignore these more recent developments in favor of the old hot Big Bang cosmology.

Given the observed cosmic expansion, mathematical extrapolation still further into the 
past - a bold move that might have little warrant, depending on how far one extrapolatesimplies that the spacetime metric was singular roughly 14,000,000,000 years ago: there was a state of infinite curvature and density, through which Einstein's equations allow no further retrodiction. Contrary to some earlier hopes still present in the 1960s, general relativistic singularity theorems show the singular behavior to be generic, not an artifact of the high symmetry assumed in homogeneous isotropic or spherically symmetric models as it is in Newtonian gravity [Wald, 1984]. If homogeneity is assumed (as it usually is, at least for the prototypical models such as Robertson-Walker), then the singularity occurs everywhere throughout all space, so it has seemed natural to speak of an origin of space (or space-time) at the singularity. However, homogeneity on scales beyond the horizon is an assumption or convention [Bondi, 1947, Layzer, 1954, McCrea, 1955, Callan et al., 1965, Klein, 1971, Feynman et al., 1995, Smoller and Temple, 2003], not an empirical fact. Indeed even the global topology of space-time is subject to worries regarding conventionality [Glymour, 1973, Malament, 1977]. Empirically we have no (direct) access to regions more distant than some billions of light years (at least apart from quantum mechanics), due to relativistic causality constraints. As a result, claims that homogeneity holds out to, say, a trillion light-years, or $10^{30}$ light-years, are not observationally well grounded. (Of course inflationary cosmology could complicate matters.) I emphasize the spatially local rather than global nature of current cosmological knowledge and hence of the singularity in anticipation of discussing the analogy between Big Bang cosmology and the time reversal of stellar gravitational collapse.

Some authors present the Big Bang singularity as potent evidence for creation in time and hence for creation ex nihilo and consequently for theism. Among the most visible proponents at present are philosopher-theologian Craig [Craig, 1979, Craig and Smith, 1993, Copan and Craig, 2004] and astrophysicist-apologist Hugh Ross [Ross, 2001, Ross, 1991], though noteworthy working physicists and astronomers have endorsed it, as well as Pope Pius XII. While Ross's works generally are not aimed at the academy, they receive endorsements by reputable physicists, cite technical papers and have some semi-popular influence. As Ross puts the issue,

[i]n Hawking's words, time itself must have a beginning. [footnote omitted] Proof of the beginning of time may rank as the most theologically significant theorem of all time, assuming validity of the general theory of relativity. [Ross, 2001, p. 102] Today it can be said that no theory of physics has ever been tested in so many different contexts and so rigorously as general relativity. The fact that general relativity has withstood all these tests so remarkably 
well implies that no basis at all remains for doubting the conclusions of the space-time theorem. [Ross, 2001, p. 107]

By contrast William Lane Craig argues more carefully and concludes more modestly. Craig formulates the Kalām cosmological argument along these lines. Craig formulates an argument along these lines:

1. Everything that begins to exist has a cause of its existence.

2. The universe began to exist.

3. Therefore the universe has a cause of its existence. [Craig, 1979, p. 63]

This argument is valid. The first premise seems true, at least on some readings of "beginning," including the one for which I argued above, namely, a first moment. The truth of the second premise, or rather, the source of warrant for the second premise, is the key question. Many theists will affirm the second premise and regard the Kalām argument as sound, but soundness is not the only relevant issue. What is the reason for affirming the second premise? Clearly one will not persuade the non-theist to accept theism (or strengthen the theist's faith with scientific support), as the argument is intended to do, if the warrant for the second premise comes wholly from ostensible divine revelation. If one accepts, say, the Bible or the Koran as divinely inspired, then one has already accepted theism and much else besides. The Big Bang singularity is an impassible barrier, blocking the extension of the past to an earlier and perhaps metrically infinite past. If the true history of the real world is characterized by such a singularity, then the world is metrically finite in age. But it is unclear whether metrical finitude of age does the work that the Kalām argument needs.

\section{Tolerance or Intolerance toward Singularities?}

The question therefore arises whether to take the singularity seriously as a feature of the real world, or to dismiss it as an artifact of incomplete physical understanding. As one sees all the time in papers on quantum gravity, most people who work on quantum gravity take for granted that the Big Bang singularity is an artifact of incomplete physical understanding and expect or hope that uniting gravity with quantum mechanics in some kind of quantum gravity will resolve the singularity into some well-defined situation that admits extrapolation to still earlier times, ad infinitum. Jayant Narlikar has persuasively deployed this point as a critique of the argument from the singularity to theism [Narlikar, 1992]. John Earman, by contrast, defends a much less widely 
held view about singularities, namely, that we should display "Tolerance for Spacetime Singularities" [Earman, 1996] and try to learn from them.

Tolerating singularities and trying to learn from them, as Earman does, is an attitude that commends itself only to GTR-exceptionalists, those who emphasize the differences between GTR and the other forces over the similarities between them. If one thinks that gravity as portrayed by GTR is importantly like other forces, then gravitational singularities are not appreciably more interesting than the singular electric field of a point charge, which simply needs to be resolved by a better theory, such as quantum electrodynamics. Thus "complete gravitational collapse [in that case is u]nimportant or at most peripheral" [Misner et al., 1973, 437]. But if gravity differs importantly from the other forces, one might conclude that "complete gravitational collapse [is c] entral to understanding the nature of matter and the universe" [Misner et al., 1973, p. 437]. Then, however, one has strong technical reasons to doubt that singularities exist as part of space-time. ${ }^{2}$ Within the Robertson-Walker cosmological space-time for $t>0$ (which is to say, always), one can 'explain' (in the fashion of Laplace's demon) each moment in terms of an earlier one [Grünbaum, 1989, Smith, 2000]. Thus there is no beginning required and premise 2 might be false, as far as physics can tell. Those who strive to 'take the lessons of relativity seriously,' as GTR-exceptionalists do, might also have reason to doubt the evolutionary space-evolving-over-time picture in favor of some inherently fourdimensional picture. If one rejects the demand for such evolutionary explanations as a hangover from Newtonian physics, then again the space-time for $t>0$ seems sufficiently self-explanatory that the singularity gives no reason to infer a Creator.

In contrast to GTR-exceptionalism, one might take the view, more common among particle physicists, that Einstein's equations describe a self-interacting massless spin 2 field, much as Maxwell's equations describe a spin 1 field and the Yang-Mills equations describe a set of self-interacting spin 1 fields [Fierz and Pauli, 1939, Rosen, 1940, Papapetrou, 1948, Gupta, 1954, Kraichnan, 1955, Feynman et al., 1995, Ogievetsky and Polubarinov, 1965, Weinberg, 1965, Sexl, 1967, Deser, 1970, Weinberg, 1972, van Nieuwenhuizen, 1973, Veltman, 1981, Fang and Fronsdal, 1979, Pitts and Schieve, 2001, Boulanger and Esole, 2002]. The distinctive features of GTR are then seen as incidental (though important) technical consequences of gravity's having spin 2 rather than spin 1 . While probably all who tolerate singularities are GTR-exceptionalists, many or most GTR-exceptionalists, such as those who work on canonical quantum gravity and loop quantum gravity, do not tolerate sin-

\footnotetext{
${ }^{2}$ One might have other reasons for doubting that singularities exist as part of space-time if one emphasizes the analogy between gravity and other forces [Pitts and Schieve, 2003].
} 
gularities.

It appears, then, that whether one is tolerant or intolerant toward singularities, it turns out that there is no first moment, because every moment is preceded by earlier moments. Thus, in the relevant sense for the Kalām argument, there is no beginning implied by physics, and so premise 2 might be false. In order for the Big Bang singularity to provide a good theistic argument, the singularity must be well enough behaved to be a real and intelligible part of space-time, and badly enough behaved that it cannot have a past. Satisfying both conditions seems difficult and unlikely to be achieved. Moreover, there are various reasons, some quite good, for not tolerating singularities, which it will be worthwhile to explore.

\section{$5 \quad$ Leibniz against Incompetent Watchmaker?}

One might think that Leibniz has provided the prototype for a good critique of the singularity argument for theism. In the Leibniz-Clarke correspondence [Alexander, 1956, pp. 11, 12], Leibniz famously argued against Isaac Newton and Samuel Clarke that God would not create the physical universe in such a way that it would break down and require repair from time to time. Leibniz took Newton's views to have just such a consequence, so if the world is analogous to a watch, then Newton's God is an incompetent watchmaker because Newton's God was required to perform miracles to restore the solar system to working order. Whereas one sometimes encounters biological dysteleology arguments, this is a physical dysteleology argument. Whether Newton and Clarke deserved this criticism need not concern us. Acceptance of the analogy between the physical world and a watch does not a rejection of miracles, for Leibniz accepted miracles of grace, while rejecting miracles posited to fix nature due to poor design [Alexander, 1956, p. 12]. If the physical world is like a watch, then it ought to be able to run forever without breaking down. But the singularity theorems of GTR show that it cannot 'run forever without breaking down.' (There is no obvious analogy to the repair of the watch.) Thus the singularity theorems arguably show that GTR demonstrates its own inadequacy, one might conclude. Because God would not build the world so incompetently, it follows that GTR is not the correct theory for describing gravitational collapse; the true theory would not yield singularities. But GTR is time-reversal invariant, and the Big Bang singularity is simply the time reversal of a specific model of gravitational collapse of a star, which uses a Robertson-Walker metric for the stellar interior [Misner et al., 1973, pp. 846-859]. Thus the Big Bang singularity would be eliminated along with the singularities of gravitational 
collapse, or so the argument goes.

This sort of argument continues to be used today, often without the explicit theism, by theoretical physicists. Thus Abhay Ashtekar, one of the dominant figures in contemporary work on quantum gravity, opened a recent review of the field with the following motivation:

Big-Bang and other singularities: It is widely believed that the prediction of a singularity, such as the big-bang of classical general relativity, is primarily a signal that the theory has been pushed beyond the domain of its validity. A key question to any quantum gravity theory, then, is: What replaces the big-bang? ... [Ashtekar, 2001]

One can find similar sentiments elsewhere very easily among workers in quantum gravity.

Leibniz might or might not have been entitled to his "God wouldn't do it that way" premise. He lived in a still Christian age and took himself to know many things by divine revelation; many contemporary physicists cannot say the same. If one is doing natural rather than revealed theology, the premise about what God would or wouldn't do seems difficult to justify. Thus Elliott Sober has recently warned against this sort of $a$ priori theological claim about what God would or ought to do [Sober, 2003, Sober, 2007]. Whereas Stephen Jay Gould seemed to think that he knew that God (if he had existed) wouldn't have made the panda's thumb as it is, Sober denies that Gould knew any such thing about the plans of the Deity. It seems especially difficult for atheists to claim to know a priori what God would or would not have done. Theists inclined toward Reformed epistemology can appeal to the causal influence of God in certain instances of belief formation [Plantinga, 2000]; whether this claim is true or not, it does not involve the theist in self-contradiction. Atheists, on the other hand, can appeal neither to the causal influence of God (on pain of self-contradiction) nor to the causal influence of the absence of God (because non-entities do not act). At best the atheist can argue that some specific God, who is ostensibly revealed in some specific holy book, would not have acted in a certain way if he had existed. Such a claim, however, must be justified in terms of the holy book in question. Most holy books have little explicit to say about space-time singularities or the panda's thumb, however, so the justification might take some doing. The situation for someone who neither affirms nor denies theism is perhaps the most interesting case, but I do not attempt to address it.

The Leibnizian intuition that God would not create a world that breaks down after a finite time has a certain appeal for physicists, but reflection suggests that it might be difficult for the argument to get traction with those not already disposed to accept it, except 
perhaps for theistic rationalists such as Leibniz. That this Leibnizian intuition appeals to physicists may reflect the fact, discussed in theories of science by Thomas Kuhn, Imre Lakatos and others [Laudan et al., 1986], that ordinary scientific progress depends upon a certain faith that things are on the right track and that progress is possible; anomalies that could serve as counterevidence are in fact typically not regarded as threatening the paradigm or hard core of the research program, but rather as opportunities to display its excellence, sooner or later, by triumphing over difficulties. The Leibnizian intuition might well be truth-conducive, but it is difficult to argue for that conclusion. If someone simply lacks this intuition, it is difficult to argue her into it. If a proponent of the singularity argument lacks this intuition, then this criticism might end in a stalemate. This phenomenon bears some resemblance to the sort of stalemate that can result in the scientific realism-antirealism debate [Kukla, 1998]. However, the sort of intellectual pessimism or strong empiricism that rejects the Leibnizian intuition, if accepted, might undermine the explanatory drive that motivates accepting premise 1 of the Kalām argument.

\section{Induction from Earlier Theories' Breakdown?}

The physics of a century ago provides some material for an inductive argument that quantum mechanics will fix the problems in Einstein's classical theory of gravity. The story of blackbody radiation and the development of quantum theory is complicated [Kuhn, 1978], much more so than the view given in modern physics textbooks, but the following selection should suffice. Just under a century ago, there were good classical theoretical arguments for the Rayleigh-Jeans law for blackbody radiation, according to which the energy density for radiation at a given frequency increased with frequency. (The Rayleigh-Jeans law was also demonstrably false empirically, but in some ways that is an irrelevant accident for present purposes.) But such a radiation law has to be wrong, because integrating over all frequencies (up to $+\infty$ ) implies that a blackbody radiates infinite power. Ordinary objects, especially black ones, approximate blackbodies, so they would radiate away their energy immediately in a blinding flash, contrary to experience. This difficulty has come to be known as the "ultraviolet catastrophe." The answer of Planck and others to the threatening inference of ultraviolet catastrophe, history shows, helped to lead to quantum mechanics. New theoretical foundations were brought in that yielded an exponentially decaying factor to counteract the Rayleigh-Jeans power law growth and thus give a convergent integral up to infinite frequency. (The exponentially decaying factor preceded widespread worries about the ultraviolet catastrophe, but previously it was motivated on 
more empirical grounds.) From a logical point of view, the ultraviolet catastrophe was a reductio ad absurdum of the classical physics underlying the Rayleigh-Jeans radiation law. The solution was new physics of a quantum kind, which averted the catastrophic infinity. In theological terms, the moment-by-moment absence of such a disaster falls under the category of general providence. A flaw in our understanding of general providence was resolved not by special providence (miracle), but by an improved understanding of general providence. Using this case and others, one might argue inductively that just as quantum mechanics resolved these problems, so it will resolve the singularity problems of GTR.

Earman insightfully discusses a similar argument to the effect that GTR contains the seeds of its own destruction [Earman, 1996], citing Kip Thorne's invocation of the Rutherford atom. However, as Earman notes, the Rutherford atom with classical electromagnetism was empirically inadequate, something that cannot be said at present for GTR's prediction of gravitational collapse. A theory that predicts disaster here and now clearly needs to be changed, if disaster is not observed. However, GTR fits the data rather well. What of its ultimate mathematical breakdown? Earman observes that if GTR is taken at face value, then there just is not some region 'beyond' the singularity that the theory fails to describe. It would seem that the supposed inductive argument relies on a metatheoretic criterion besides empirical adequacy to determine that GTR breaks down, one that strongly resembles the Leibnizian intuition previously discussed. Thus "[t]he analogy with the Rutherford atom is not apt." [Earman, 1996] So this inductive argument will not persuade those who are not persuaded by the Leibnizian intuition.

\section{Stellar Collapse Implies Theistic Destroyer}

One might wonder why theological significance should be ascribed to the Big Bang singularity, but not to other physical singularities. This worry takes its most acute form when one considers the similarity of the Big Bang cosmology to the time reverse of the gravitational collapse of a star to a black hole with a central singularity. Assuming homogeneous matter distributions, both Big Bang cosmology and stellar gravitational collapse use the Robertson-Walker space-time metric in the matter-filled region [Misner et al., 1973, pp. 846-859]. If one chooses a Big Bang model with a bounded matter distribution, as one certainly may [Bondi, 1947, Layzer, 1954, McCrea, 1955, Callan et al., 1965, Klein, 1971, Feynman et al., 1995, Smoller and Temple, 2003], then both the Big Bang and gravitational collapse have a Robertson-Walker interior matched to a Schwarzschild exterior. 
The main differences are the direction of time and the distance scale. The distance scale does not seem important for present purposes. If the Big Bang strongly indicates that there exists a God who created the universe, do formally similar time-reversed events such as the gravitational collapse of stars to form black holes with singularities imply that there exists a God who supernaturally destroys (annihilates, ceases to uphold) the interiors of stars? This is a rather surprising conclusion. While Christianity portrays God as creating, sustaining and redeeming the world, the idea of God's absolutely annihilating either the physical world as a whole or a bit here and there from time to time, appears to be a novelty or certainly a rarity in Christian theology. Of course rocks, buildings and animals perish from time to time, but their physical remains persist, which is precisely what does not happen here. Orthodoxy has held that even those who 'perish' in hell persist forever, body and soul, albeit in a miserable condition. Given the divine policy of upholding the universe after creating it, for God to stop upholding, say, a star that collapses to a black hole, would be a miracle. Thus, whatever asymmetry of time might exist in relation to causation, does not help to avoid the conclusion that stellar gravitational collapse terminates in a miracle. The proponent of the Big Bang argument therefore needs to explain why the termination of stellar gravitational collapse is not a miracle, or why such a miracle is not absurd. It is somewhat comforting that God, on the view in question, would only annihilate regions of high density, perhaps typically surrounded by an event horizon. Thus there is plenty of warning so that we may avoid these episodes of annihilation, and the farmer who stores wheat in a silo need not fear the annihilation of his wheat. Such a divine policy suffices for practical purposes. However, positing miracles without any discernible divine purpose appears to have been rejected by all serious people at all times, however friendly some of them were to miracles which had some specifiable divine purpose - controversies of much weight in the 16th and 17th centuries. It appears that one who argues from the Big Bang singularity is committed to a novel and unreasonable view that God sometimes supernaturally destroys collapsed stars. If GTR were the only possible theory to describe the divine governance of the world in matters gravitational, perhaps one could manage to accept that conclusion. But given the underdetermination of theories by data, the inference that God supernaturally destroys stars appears to be a reductio ad absurdum of the Big Bang singularity argument for theism. 


\section{Stacking the Deck for General Relativity}

There is a misleadingly persuasive move made by proponents of the Big Bang argument, stacking the deck in favor of general relativity and thus of Big Bang cosmology against nonsingular rivals. The move is generally not made explicitly and in detail, so what follows is a reconstruction of the reasoning process that would underlie any good argument in the vicinity. It is often suggested that potentially nonsingular rivals to general relativity are speculative, whereas general relativity is well confirmed, so general relativity and its retrodiction of the Big Bang singularity ought to be accepted as the default view that challengers need to overcome with better empirical results. There is a grain of truth in this claim: some or perhaps many of the theories or models actually proposed as rivals to the Big Bang in the more speculative literature do not form part of a well-tested theory that is known to reproduce the empirical successes of general relativity. Thus some of these challengers might be refuted by data already in hand, should someone think to do the necessary calculations and apply the relevant empirical data to the challengers. Were the physics community's (or its members') physical knowledge closed under entailment, such would-be challengers would be refuted even before publication; alas, human finitude

intrudes. However, the grain of truth in this objection sometimes conceals the grain of falsehood that it also contains. Though not entirely trivial, it is possible to construct theories that reproduce the empirical successes of general relativity in all tested regimes to date, but which differ in the ultra-strong field regime relevant to Big Bang cosmology. Granting the success of weak- and medium-field tests of general relativity involving light bending, gravitational redshifting, time delay, and the like, why think that general relativity, rather than one of its (perhaps as-yet unproposed) competitors that fits the data currently in hand, is the right extrapolation?

\section{Quantum Gravity Tends to Resolve Singularities}

The need to reconcile gravity and quantum mechanics all but proves that there exists a consistent theory of gravity that matches general relativity in some classical limit, but which differs from it in regimes when dimensional arguments suggest that quantum effects should be large. There might well be many such theories of quantum gravity. The works of Ashtekar and collaborators, such as [Ashtekar et al., 2006b, Ashtekar et al., 2006a], and Bojowald and collaborators, such as [Bojowald, 2001a, Bojowald, 2001b, Bojowald, 2002, Bojowald and Hinterleitner, 2002, Bojowald and Kagan, 2006], provide good evidence that the Big Bang singularity does not occur in loop quantum gravity (but see ([Cartin and Khanna, 2005]). 
Loop quantum gravity is a part of the modern nonperturbative canonical quantum gravity project [Pullin, 2003], which began began in the 1980s when Abhay Ashtekar proposed new variables that helped to resolve long-standing problems faced when using the older metric variables.

Moreover, a quantum theory of gravity is likely to differ considerably from GTR precisely in the ultra-strong field regime of the hot dense 'early' universe, where the Big Bang arguer relies essentially on GTR! Thus Robert Wald writes in a standard graduate textbook on GTR: "Of course, at the extreme conditions very near the big bang singularity one expects that quantum effects will become important, and the predictions of classical general relativity are expected to break down." [Wald, 1984, p. 100] In such a context, curvatures comparable to the inverse square of the Planck length arise, so neglected quantum terms should be large and the classical theory becomes a bad approximation. In addition to the modern canonical quantization program, one should also keep an eye on string theory as tending to resolve singularities [Gasperini and Veneziano, 2003], not to mention various classical proposals that alter the dynamics in the strong field regime.

Lawrence Sklar once asked "Do Unborn Hypotheses Have Rights?" [Sklar, 1985]. Clearly they do in the present context. As Bas van Fraassen has noted in the context of criticizing inference to the best explanation, "[w] can watch no contest of the theories we have so painfully struggled to formulate, with those no one has proposed. So our selection may well be the best of a bad lot." [van Fraassen, 1989, p. 143] P. Kyle Stanford argues that the problem of unconceived alternatives is an even more serious problem for scientific realism than are more commonly discussed worries [Stanford, 2006, Stanford, 2001]. Once the rights of unborn theories are respected, the default status allegedly held by general relativity and hence of Big Bang cosmology as described by GTR near the singularity disappears. In the ultra-strong field regime of the hot dense so-called 'early universe' (to use a term that presupposes the Big Bang singularity), GTR is just another speculation among many. Is one truly rationally compelled, or even rationally encouraged, to accept an infinite extrapolation from a curve that fits the data in some finite region? Surely not. Sophisticated defenders of scientific realism now admit that different parts of a scientific theory are supported to different degrees by the theory's empirical confirmation [Psillos, 1999, chs. 5, 6]. In the context of GTR, one should recognize that the theory's success in weak- and medium-strength gravitational fields provides little support for the theory's accuracy in strong gravitational fields such as near the Big Bang. But there is no reason to restrict the competitors of GTR to theories that someone on Earth has already proposed. The relevant set of competitors for GTR includes the set of theories 
that agree with GTR on all experiments to date, whether already entertained on Earth or not. This set might be infinite, might well be large, likely contains several members, and almost certainly has at least one member, a quantum theory of gravity. The set most likely has at least one member that resolves the singularities of GTR. Thus in the strong-field regime, it is not at all clear why one should take GTR seriously.

\section{Vicious God-of-the-Gaps Character}

Some of the argumentation above resembles typical worries about 'God-of-the-gaps' apologetic arguments [Saunders, 2002]. According to such worries, there is a long history of appeals to special divine action to explain certain phenomena, but later natural explanations for such phenomena appeared, making the appeal to special divine action unnecessary. Making an induction over this history, one is supposed to learn the lesson not to appeal to special divine action in such cases, lest one make religion look foolish yet again when the gaps close. It has been argued recently that worries about God-of-the-gaps arguments are overstated philosophically [Ratzsch, 2001, Snoke, 2001, Larmer, 2002]; Del Ratzsch notes, for example, that the argument form is valid. These worries might also be overstated historically. Are they part of the same complex of overstatement and distortion as the Huxley-Draper-White science-religion warfare thesis [White, 1896, Lindberg and Numbers, 1986, Brooke, 1991] and its subtheses such as the Copernicus-dethroned-Earth thesis [Danielson, 2001] and the medievals-believed-in-a-flat Earth thesis [Russell, 1991]? To my knowledge, the definitive history of God-of-the-gaps arguments and their critiques has yet to be written.

Regarding the argument from the Big Bang singularity to theism, physicist Chris Isham notes "its obvious susceptibility to the 'God of the gaps' syndrome, in which God is relegated to filling in the blanks of an otherwise complete scientific theory" [Isham, 1997, p. 378]. Even if one concedes that some gaps arguments for theism might be good arguments, it remains clear that the Big Bang singularity argument is a bad argument from gaps to God. That is clear because there is no non-conventional relevant difference between the Big Bang singularity and stellar gravitational collapse. The latter surely has no theological significance. Instead it indicates an incomplete understanding of physics and hence of God's ordinary providence (given theism). Thus this case is clearly the sort that should be addressed theologically in ordinary providence rather than special (miraculous) providence. It is not implausible that some singularity-wielding theistic apologists will be tempted to resist scientific progress in the form of a new quantum 
theory of gravity in order to maintain an apologetic strategy in which they have invested. Thus theistic apologists ought to be wary of arguing from the singularity to creation and then to theism. Here I have assumed an intolerant attitude toward singularities. If one does tolerate singularities, then every moment of the Big Bang model $(t>0)$ is preceded by earlier moments and again there is no call for extramundane explanation.

\section{Fluctuating and Inaccessible Warrant}

A related problem with arguing from the Big Bang to creation in time is that such arguments depend crucially on various highly technical premises which most people cannot even entertain, much less evaluate. It follows that the vast majority of people, even educated ones, simply are not entitled to beliefs on the matter, apart from relying on the testimony of experts. But most people, even most educated people, cannot even reliably identify relevant experts. Most astronomers and physicists are not relevant experts, though they might well write popular books and make statements to the media on such issues. Supposing that one manages to identify relevant experts, the problem remains that their expert opinions will or should vary rather rapidly with the winds and waves of research fortune. But should theology be affected much by the validity of Weak or Averaged Null Energy Condition assumed for singularity theorems? It is now known that quantum field theory violates the local classical energy conditions such as the Weak Energy Condition, though apparently quantum field theory still satisfies certain averaged energy conditions: energy density can be negative here and there, but not for very long and only with greater compensation of positive energy nearby. Nonminimally coupled scalar fields violate energy conditions [Ford, 2003, Ford and Roman, 2001, Barcelo and Visser, 2002], as does massive gravity [Visser, 1998]. Are journalists, sociologists, homemakers and truck drivers supposed to accept an argument whose premises are so technical that they cannot understand them, and so unstable that they could prove false in the next issue of Physical Review D? It is not clear why. Should the strength of one's faith depend on which factor ordering for the Hamiltonian constraint is correct in quantum gravity? Martin Bojowald fairly recently wrote in a paper's abstract [Bojowald, 2002]:

Because of genuinely quantum geometrical effects the classical singularity is absent in those models in the sense that the evolution does not break down there, contrary to the classical situation where space time is inextendible. This effect is generic and does not depend on matter violating energy conditions, but it does depend on the factor ordering of the Hamiltonian constraint. 
Most people have no idea what that means and thus no idea what sort of plausibility to assign a particular factor ordering of the Hamiltonian constraint. If some of them believed in God because of the Big Bang singularity argument, must they now be able to refute Bojowald's choice of factor ordering in order to maintain that belief rationally? Even if the singularity argument could not be decisively refuted, could it establish theism to a significant degree for anyone besides the few dozens of people expert in factor ordering in quantum gravity? It is unclear how. Could even the experts' judgments rationally remain stable enough to serve religious faith well? Probably not. But perhaps the choice of factor ordering is not so important after all [Date and Hossain, 2005]; the fluctuations based on detailed technical premises continue. ${ }^{3}$ If the singularity argument for theism does provide any warrant for theism, that warrant fluctuates wildly for experts and cannot be assessed by the laity.

Perhaps neither the singularity argument nor any other argument is the basis for religious faith. Craig holds to something like Reformed epistemology [Plantinga, 2000] (private correspondence and [Cowan et al., 2000]), which posits the work of God the Holy Spirit as a causally reliable process that produces and sustains religious faith. Thus the religious believer does not need to read and understand every relevant paper in Physical Review D to maintain theistic belief rationally. Perhaps this is the correct way to understand the warrant for theistic belief. But if the point of making the singularity argument is to provide an argument that not merely in fact persuades some people of theism, but rationally ought to persuade them, then Reformed epistemology is simply irrelevant to the question at hand. The fluctuating (for the experts) and inaccessible (for non-experts) character of such warrant (if any) as an argument from the singularity to theism is another reason that theistic apologists ought to abandon this strategy.

\section{Conclusion}

Given the many difficulties involved in arguing from Big Bang cosmology to creation in time, it is encouraging that Pope John Paul II has suggested caution:

\footnotetext{
${ }^{3}$ The point having been made, perhaps a brief explanation of the factor ordering problem is appropriate [Komar, 1979]. In classical physics, the Hamiltonian description involves various products of coordinates $q$ and momenta $p$. In some theories, every term is just a power of $q$ or of $p$, but not both. But what if there is a term involving powers of both $q$ and $p$, as is true in Einstein's theory of gravity? Then the order in which they are written, such as $p^{2} q^{2}$ or $q^{2} p^{2}$ or $q p p q$ for example, though of no importance classically, is of some importance in quantum theory.
} 
... some theologians, at least, should be sufficiently well-versed in the sciences to make authentic and creative use of the resources that the best-established theories may offer them. Such an expertise would prevent them from making uncritical and overhasty use for apologetic purposes of such recent theories as that of the "Big Bang" in cosmology. [John Paul II, 1997, pp. M11, M12]

This papal call for caution in apologetic use of Big Bang cosmology compares favorably with the rather less cautious remarks of Pope Pius XII in 1951 that caused concern for Lemaître and McMullin [McMullin, 1981a, Kragh, 2004].

\section{References}

[Agnese and Wataghin, 1971] Agnese, A. G. and Wataghin, A. (1971). Scale factor in cosmological homogeneous models. Lettere al Novo Cimento, 1:857.

[Alexander, 1956] Alexander, H. G., editor (1956). The Leibniz-Clarke Correspondence. Manchester University, Manchester.

[Anderson, 1967] Anderson, J. L. (1967). Principles of Relativity Physics. Academic, New York.

[Ashtekar, 2001] Ashtekar, A. (2001). Quantum geometry and gravity: Recent advances. gr-qc/0112038. report of the plenary talk at the 16th International Conference on General Relativity and Gravitation, held at Durban, S. Africa in July 2001.

[Ashtekar et al., 2006a] Ashtekar, A., Pawlowski, T., and Singh, P. (2006a). Quantum nature of the Big Bang: An analytical and numerical investigation. Physical Review D, 73:124038. gr-qc/0604013v3.

[Ashtekar et al., 2006b] Ashtekar, A., Pawlowski, T., and Singh, P. (2006b). Quantum nature of the Big Bang: Improved dynamics. Physical Review D, 74:084003. grqc/0607039v2.

[Barcelo and Visser, 2002] Barcelo, C. and Visser, M. (2002). Twilight for the energy conditions? International Journal for Modern Physics D, 11:1553. gr-qc/0205066; honorable mention by Gravity Research Foundation.

[Bojowald, 2001a] Bojowald, M. (2001a). Absence of singularity in loop quantum cosmology. Physical Review Letters, 86:5227. gr-qc/0102069.

[Bojowald, 2001b] Bojowald, M. (2001b). The inverse scale factor in isotropic quantum geometry. Physical Review D, 64:084018. gr-qc/0105067. 
[Bojowald, 2002] Bojowald, M. (2002). Isotropic loop quantum cosmology. Classical and Quantum Gravity, 19:2717. gr-qc/0202077.

[Bojowald and Hinterleitner, 2002] Bojowald, M. and Hinterleitner, F. (2002). Isotropic loop quantum cosmology with matter. Physical Review D, 66:104003. gr-qc/0207038.

[Bojowald and Kagan, 2006] Bojowald, M. and Kagan, M. (2006). Singularities in isotropic non-minimal scalar field models. Classical and Quantum Gravity, 23:4983. gr-qc/0604105.

[Bondi, 1947] Bondi, H. (1947). Spherically symmetrical models in general relativity. Monthly Notices of the Royal Astronomical Society, 107:410. NASA ADS at Harvard.

[Boulanger and Esole, 2002] Boulanger, N. and Esole, M. (2002). A note on the uniqueness of $\mathrm{D}=4, \mathrm{~N}=1$ supergravity. Classical and Quantum Gravity, 19:2107. grqc/0110072v2.

[Brooke, 1991] Brooke, J. H. (1991). Science and Religion: Some Historical Perspectives. Cambridge University, Cambridge.

[Buchert, 2007] Buchert, T. (2007). Dark energy from structure: A status report. General Relativity and Gravitation, forthcoming. gr-qc/0707.2153.

[Buchert and Carfora, 2002] Buchert, T. and Carfora, M. (2002). Regional averaging and scaling in relativistic cosmology. Classical and Quantum Gravity, 19:6109. grqc/0210037v2.

[Callan et al., 1965] Callan, C., Dicke, R. H., and Peebles, P. J. E. (1965). Cosmology and Newtonian mechanics. American Journal of Physics, 33:105.

[Cartin and Khanna, 2005] Cartin, D. and Khanna, G. (2005). Absence of pre-classical solutions in Bianchi I loop quantum cosmology. Physical Review Letters, 94:111302. gr-qc/0501016v2.

[Catena et al., 2006] Catena, R., Pietroni, M., , and Scarabello, L. (2006). Einstein and Jordan reconciled: A frame-invariant approach to scalar-tensor cosmology. www.arxiv.org, astro-ph/0604492.

[Cattoen and Visser, 2007] Cattoen, C. and Visser, M. (2007). Cosmography: Extracting the Hubble series from the supernova data. www.arxiv.org, gr-qc/0703122.

[Coley and Pelavas, 2006] Coley, A. A. and Pelavas, N. (2006). Averaging spherically symmetric spacetimes in general relativity. Physical Review D, 74:087301.

[Copan and Craig, 2004] Copan, P. and Craig, W. L. (2004). Creation out of Nothing: A Biblical, Philosophical and Scientific Exploration. Baker Academic, Grand Rapids. 
[Cowan et al., 2000] Cowan, S. B., Craig, W. L., Habermas, G. R., Feinberg, P. D., Frame, J. M., and Clark, K. J. (2000). Five Views on Apologetics. Zondervan, Grand Rapids.

[Craig, 1979] Craig, W. L. (1979). The Kalām Cosmological Argument. Barnes and Noble, New York.

[Craig and Smith, 1993] Craig, W. L. and Smith, Q. (1993). Theism, Atheism, and Big Bang Cosmology. Clarendon, Oxford.

[Danielson, 2001] Danielson, D. R. (2001). The great Copernican cliché. American Journal of Physics, 69:1029.

[Date and Hossain, 2005] Date, G. and Hossain, G. M. (2005). Genericness of a big bounce in isotropic loop quantum cosmology. Physical Review Letters, 94:011302. grqc/0407074.

[Deser, 1970] Deser, S. (1970). Self-interaction and gauge invariance. General Relativity and Gravitation, 1:9. gr-qc/0411023v2.

[Dzhunushaliev and Schmidt, 2000] Dzhunushaliev, V. and Schmidt, H.-J. (2000). New vacuum solutions of conformal Weyl gravity. Journal of Mathematical Physics, 41:3007.

[Earman, 1996] Earman, J. (1996). Tolerance for spacetime singularities. Foundations of Physics, 26:623.

[Earman and Mosterin, 1999] Earman, J. and Mosterin, J. (1999). A critical look at inflationary cosmology. Philosophy of Science, 66:1.

[Ellis, 1984] Ellis, G. F. R. (1984). Relativistic cosmology: Its nature, aims and problems. In Bertotti, B., de Felice, F., and Pascolini, A., editors, General Relativity and Gravitation: Invited Papers and Discussion Reports of the 10th International Conference on General Relativity and Gravitation, Padua, July 3-8, 1983. D. Reidel, Dordrecht.

[Fang and Fronsdal, 1979] Fang, J. and Fronsdal, C. (1979). Deformations of gauge groups. Gravitation. Journal of Mathematical Physics, 20:2264.

[Faraoni and Nadeau, 2007] Faraoni, V. and Nadeau, S. (2007). (Pseudo)issue of the conformal frame revisited. Physical Review D, 75:023501. gr-qc/0612075v1.

[Feynman et al., 1995] Feynman, R. P., Morinigo, F. B., Wagner, W. G., Hatfield, B., Preskill, J., and Thorne, K. S. (1995). Feynman Lectures on Gravitation. AddisonWesley, Reading, Mass. Original by California Institute of Technology, 1963.

[Fiedler and Schimming, 1980] Fiedler, B. and Schimming, R. (1980). Exact solutions of the Bach field equations of general relativity. Reports on Mathematical Physics, 17:15. 
[Fierz and Pauli, 1939] Fierz, M. and Pauli, W. (1939). On relativistic wave equations for particles of arbitrary spin in an electromagnetic field. Proceedings of the Royal Society (London) A, 173:211.

[Ford, 2003] Ford, L. H. (2003). The classical singularity theorems and their quantum loopholes. International Journal of Theoretical Physics, 42:1219. gr-qc/0301045.

[Ford and Roman, 2001] Ford, L. H. and Roman, T. A. (2001). Classical scalar fields and the generalized second law. Physical Review D, 64:024023. gr-qc/0009076.

[Gasperini and Veneziano, 2003] Gasperini, M. and Veneziano, G. (2003). The pre-big bang scenario in string cosmology. Physics Reports, 373:1-212. hep-th/0207130.

[Glymour, 1973] Glymour, C. (1973). Topology, cosmology, and convention. In Suppes, P., editor, Space, Time, and Geometry, page 193. D. Reidel, Dordrecht.

[Grünbaum, 1973] Grünbaum, A. (1973). Philosophical Problems of Space and Time. Reidel, Dordrecht, second edition.

[Grünbaum, 1989] Grünbaum, A. (1989). The pseudo-problem of creation in physical cosmology. Philosophy of Science, 56:373.

[Gupta, 1954] Gupta, S. N. (1954). Gravitation and electromagnetism. Physical Review, 96:1683.

[Helm, 1997] Helm, P. (1997). Eternal creation: The doctrine of the two standpoints. In Gunton, C. E., editor, The Doctrine of Creation: Essays in Dogmatics, History and Philosophy. T\&T Clark, Edinburgh.

[Hyman and Walsh, 1973] Hyman, A. and Walsh, J. J. (1973). Philosophy in the Middle Ages: The Christian, Islamic, and Jewish Traditions. Hackett, Indianapolis, second edition.

[Isham, 1997] Isham, C. J. (1997). Creation of the universe as a quantum process. In Russell, R. J., Stoeger, W. R., and Coyne, G. V., editors, Physics, Philosophy, and Theology: A Common Quest for Understanding. Vatican Observatory, Vatican City State/University of Notre Dame, third edition.

[John Paul II, 1997] John Paul II (1997). To the Reverend George V. Coyne, S.J., Director of the Vatican Observatory, 1 June 1988. In Russell, R. J., Stoeger, W. R., and Coyne, G. V., editors, Physics, Philosophy, and Theology: A Common Quest for Understanding. Vatican Observatory, Vatican City State/University of Notre Dame, third edition.

[Kaloper and Olive, 1998] Kaloper, N. and Olive, K. A. (1998). Singularities in scalartensor cosmologies. Physical Review D, 57:811. hep-th/9708008. 
[Kazanas and Mannheim, 1991] Kazanas, D. and Mannheim, P. D. (1991). General structure of the gravitational equations of motion in conformal Weyl gravity. Astrophysical Journal Supplement Series, 76(2):431.

[Klein, 1971] Klein, O. (1971). Arguments concerning relativity and cosmology. Science, 171:339.

[Komar, 1979] Komar, A. (1979). Consistent factor ordering of general-relativistic constraints. Physical Review D, 20:830.

[Kragh, 2004] Kragh, H. (2004). Matter and Spirit in the Universe: Scientific and Religious Preludes to Modern Cosmology. Imperial College, London.

[Kraichnan, 1955] Kraichnan, R. H. (1955). Special-relativistic derivation of generally covariant gravitation theory. Physical Review, 98:1118.

[Kuhn, 1978] Kuhn, T. S. (1978). Black-body Theory and the Quantum Discontinuity, 1894-1912. Clarendon, Oxford.

[Kukla, 1998] Kukla, A. (1998). Studies in Scientific Realism. Oxford University Press, New York.

[Larmer, 2002] Larmer, R. (2002). Is there anything wrong with "God of the gaps" reasoning? International Journal for Philosophy of Religion, 52:129.

[Laudan et al., 1986] Laudan, L., Donovan, A., Laudan, R., Barker, P., Brown, H., Leplin, J., Thagard, P., , and Wykstra, S. (1986). Scientific change: Philosophical models and historical research. Synthese, 69:141.

[Layzer, 1954] Layzer, D. (1954). On the significance of Newtonian cosmology. The Astronomical Journal, 59:268. NASA ADS at Harvard.

[Levy-Leblond, 1990] Levy-Leblond, J.-M. (1990). Did the Big Bang begin? American Journal of Physics, 58:156.

[Lindberg and Numbers, 1986] Lindberg, D. C. and Numbers, R. L., editors (1986). God $\mathscr{G}$ Nature: Historical Essays on the Encounter between Science and Religion. University of California, Berkeley.

[Magnano and Sokolowski, 1994] Magnano, G. and Sokolowski, L. M. (1994). On physical equivalence between nonlinear gravity theories. Physical Review D, 50:5039. grqc/9312008.

[Malament, 1977] Malament, D. (1977). Observationally indistinguishable space-times. In Earman, J., Glymour, C., and Stachel, J., editors, Foundations of Space-Time Theories. University of Minnesota, Minneapolis. 
[McCrea, 1955] McCrea, W. H. (1955). On the significance of Newtonian cosmology. The Astronomical Journal, 60:271. NASA ADS at Harvard.

[McMullin, 1981a] McMullin, E. (1981a). How should cosmology relate to theology? In Peacocke, A. R., editor, The Sciences and Theology in the Twentieth Century. University of Notre Dame, Notre Dame, Indiana.

[McMullin, 1981b] McMullin, E. (1981b). Is philosophy relevant to cosmology? American Philosophical Quarterly, 18:177.

[Misner et al., 1973] Misner, C., Thorne, K., and Wheeler, J. A. (1973). Gravitation. Freeman, New York.

[Misner, 1969] Misner, C. W. (1969). Absolute zero of time. Physical Review A, 186:1328.

[Morriston, 2003] Morriston, W. (2003). Must metaphysical time have a beginning? Faith and Philosophy, 20:288.

[Narlikar, 1992] Narlikar, J. V. (1992). The concepts of "beginning" and "creation" in cosmology. Philosophy of Science, 59:361.

[Ogievetsky and Polubarinov, 1965] Ogievetsky, V. I. and Polubarinov, I. V. (1965). Interacting field of spin 2 and the Einstein equations. Annals of Physics, 35:167.

[Papapetrou, 1948] Papapetrou, A. (1948). Einstein's theory of gravitation and flat space. Proceedings of the Royal Irish Academy A, 52:11.

[Pitts and Schieve, 2001] Pitts, J. B. and Schieve, W. C. (2001). Slightly bimetric gravitation. General Relativity and Gravitation, 33:1319. gr-qc/0101058v3.

[Pitts and Schieve, 2003] Pitts, J. B. and Schieve, W. C. (2003). Nonsingularity of flat Robertson-Walker models in the special relativistic approach to Einstein's equations. Foundations of Physics, 33:1315. gr-qc/0406103.

[Pius XII, 1952] Pius XII (1952). Modern science and the existence of God. The Catholic Mind, 50(March):182. Address to the Pontifical Academy of Science, November 22, 1951.

[Plantinga, 2000] Plantinga, A. (2000). Warranted Christian Belief. Oxford University, New York.

[Polkinghorne, 1994] Polkinghorne, J. (1994). Science and Christian Belief. SPCK, London.

[Psillos, 1999] Psillos, S. (1999). Scientific Realism: How Science Tracks Truth. Routledge, London. 
[Pullin, 2003] Pullin, J. (2003). Canonical quantization of general relativity: the last 18 years in a nutshell. In Novello, M., editor, Proceedings of the Xth Brazilian School on Cosmology and Gravitation: AIP Conference Proceedings 668, page 141. American Institute of Physics.

[Quinn, 1993] Quinn, P. L. (1993). Creation, conservation, and the big bang. In Earman, J., Janis, A. I., Massey, G. J., and Rescher, N., editors, Philosophical Problems of the Internal and External Worlds: Essays on the Philosophy of Adolf Grünbaum, page 589. University of Pittsburgh and Universitätsverlag Konstanz, Pittsburgh and Konstanz.

[Ratzsch, 2001] Ratzsch, D. (2001). Nature, Design, and Science: The Status of Design in Natural Science. SUNY, Albany.

[Rosen, 1940] Rosen, N. (1940). General relativity and flat space. I., II. Physical Review, $57: 147,150$.

[Ross, 1991] Ross, H. (1991). The Fingerprint of God. Promise, Orange, California, second edition.

[Ross, 2001] Ross, H. (2001). The Creator and the Cosmos: How the Greatest Scientific Discoveries of the Century Reveal God. NavPress, Colorado Springs, third edition.

[Russell, 1991] Russell, J. B. (1991). Inventing the Flat Earth: Columbus and Modern Historians. Praeger, Westport, CT.

[Russell, 1996a] Russell, R. J. (1996a). Finite creation without a beginning. In Russell, R. J., Murphey, N., and Isham, C. J., editors, Quantum Cosmology and the Laws of Nature: Scientific Perspectives on Divine Action, page 291. Vatican Observatory, Vatican City State, and the Center for Theology and the Natural Sciences, Berkeley/University of Notre Dame, second edition.

[Russell, 1996b] Russell, R. J. (1996b). $t=0$ : Is it theologically significant? In Richardson, W. M. and Wildman, W. J., editors, Religion \& Science: History, Method, Dialogue. Routledge, New York.

[Santiago and Silbergleit, 2000] Santiago, D. I. and Silbergleit, A. S. (2000). On the energy-momentum tensor of the scalar field in scalar-tensor theories of gravity. General Relativity and Gravitation, 32:565. gr-qc/9904003.

[Saunders, 2002] Saunders, N. (2002). Divine Action \&3 Modern Science. CUP, Cambridge.

[Schimming and Schmidt, 1990] Schimming, R. and Schmidt, H.-J. (1990). On the history of fourth order metric theories of gravitation. NTM Schriftenreihe für Geschichte der Naturwissenschaften, Technik und Medizin, 27:41. gr-qc/0412038. 
[Schücking, 1967] Schücking, E. L. (1967). Cosmology. In Ehlers, J., editor, Relativity Theory and Astrophysics: 1. Relativity and Cosmology, page 218. American Mathematical Society, Providence, Rhode Island.

[Sexl, 1967] Sexl, R. U. (1967). Theories of gravitation. Fortschritte der Physik, 15:269.

[Sklar, 1985] Sklar, L. (1985). Do unborn hypotheses have rights? In Philosophy and Spacetime Physics. University of California, Berkeley.

[Smith, 1992] Smith, Q. (1992). A Big Bang cosmological argument for God's nonexistence. Faith and Philosophy, 9:217.

[Smith, 2000] Smith, Q. (2000). Problems with John Earman's attempt to reconcile theism with General Relativity. Erkenntnis, 52:1.

[Smoller and Temple, 2003] Smoller, J. and Temple, B. (2003). Shock-wave cosmology inside a black hole. Proceedings of the National Academy of Sciences of the United States of America, 100:11216.

[Snoke, 2001] Snoke, D. (Sept. 2001). In favor of God-of-the-gaps reasoning. Perspectives on Science \&3 Christian Faith, 53:152. http://www.asa3.org/ASA/PSCF/2001/PSCF901Snoke.html.

[Sober, 2003] Sober, E. (2003). The design argument. In Manson, N. A., editor, God and Design: The Teleological Argument and Modern Science. Routledge, London.

[Sober, 2007] Sober, E. (2007). What is wrong with intelligent design? Quarterly Review of Biology, 82:3-8. http://philosophy.wisc.edu/sober/what's

[Sotiriou et al., 2007] Sotiriou, T. P., Faraoni, V., and Liberati, S. (2007). Theory of gravitation theories: A no-progress report. www.arxiv.org, gr-qc/0707.2748.

[Stanford, 2001] Stanford, P. K. (2001). Refusing the devil's bargain: What kind of underdetermination should we take seriously? Philosophy of Science, 68:S1-S12. PSA 2000 Proceedings.

[Stanford, 2006] Stanford, P. K. (2006). Exceeding Our Grasp: Science, History, and the Problem of Unconceived Alternatives. Oxford University, New York.

[Thijssen, 1998] Thijssen, J. M. M. H. (1998). Eternity of the world, medieval views of. In Craig, E., editor, Routledge Encyclopedia of Philosophy. Routledge, London. retrieved November 13, 2004, from http://www.rep.routledge.com/article/B039.

[van Fraassen, 1989] van Fraassen, B. (1989). Laws and Symmetry. Clarendon Press, Oxford. 
[van Nieuwenhuizen, 1973] van Nieuwenhuizen, P. (1973). On ghost-free tensor Lagrangians and linearized gravitation. Nuclear Physics B, 60:478.

[Veltman, 1981] Veltman, M. (1981). Quantum theory of gravitation. In Les Houches XXVIII 1975: Methods in Field Theory, page 265. North-Holland, Amsterdam.

[Visser, 1998] Visser, M. (1998). Mass for the graviton. General Relativity and Gravitation, 30:1717. gr-qc/9705051v2.

[Wald, 1984] Wald, R. M. (1984). General Relativity. University of Chicago, Chicago.

[Weinberg, 1965] Weinberg, S. (1965). Photons and gravitons in perturbation theory: Derivation of Maxwell's and Einstein's equations. Physical Review, 138:B988.

[Weinberg, 1972] Weinberg, S. (1972). Gravitation and Cosmology. Wiley, New York.

[Weinstein, 1996] Weinstein, S. (1996). Strange couplings and space-time structure. Philosophy of Science, 63S:S63. Proceedings of the 1996 Biennial Meetings of the Philosophy of Science Association. Part I: Contributed Papers.

[White, 1896] White, A. D. (1896). A History of the Warfare of Science with Theology in Christendom. reprint Prometheus, Buffalo, 1993.

[Will, 1993] Will, C. M. (1993). Theory and Experiment in Gravitational Physics. Cambridge University, Cambridge, revised edition. 